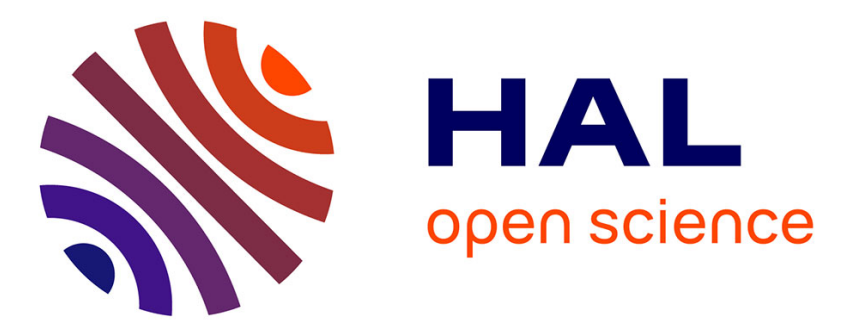

\title{
On the Complementarity of an Ontology and a Nearest Neighbour Classifier for Remotely Sensed Image Interpretation
}

\author{
Sébastien Derivaux, Nicolas Durand, Cédric Wemmert
}

\section{To cite this version:}

Sébastien Derivaux, Nicolas Durand, Cédric Wemmert. On the Complementarity of an Ontology and a Nearest Neighbour Classifier for Remotely Sensed Image Interpretation. International Geoscience and Remote Sensing Symposium (IGARSS 2007), 2007, Barcelona, Spain. pp.3983 - 3986 , 10.1109/IGARSS.2007.4423093 . hal-01463171

\section{HAL Id: hal-01463171 \\ https://hal.science/hal-01463171}

Submitted on 1 Nov 2017

HAL is a multi-disciplinary open access archive for the deposit and dissemination of scientific research documents, whether they are published or not. The documents may come from teaching and research institutions in France or abroad, or from public or private research centers.
L'archive ouverte pluridisciplinaire HAL, est destinée au dépôt et à la diffusion de documents scientifiques de niveau recherche, publiés ou non, émanant des établissements d'enseignement et de recherche français ou étrangers, des laboratoires publics ou privés. 


\title{
On the Complementarity of an Ontology and a Nearest Neighbour Classifier for Remotely Sensed Image Interpretation
}

\author{
Sébastien Derivaux, Nicolas Durand and Cédric Wemmert \\ LSIIT - CNRS - University Louis Pasteur - UMR 7005 \\ Pôle API, Bd Sébastien Brant - 67412 Illkirch, France \\ Email: \{derivaux,durand,wemmert\}@ 1siit.u-strasbg.fr
}

\begin{abstract}
Automatic image interpretation could be achieved by first performing a segmentation of the image, i.e. aggregating similar pixels to form regions, then use a region-based classification. This paper presents two region-based classifications, namely a supervised classification and an ontology-based classification and discuss their pros and cons. As they are complementary, we propose to combine these two approaches. Results shown that the presented method is relevant.
\end{abstract}

\section{INTRODUCTION}

Automatic interpretation of remote sensing images becomes an increasingly active domain. Sensors are now capable to obtain images with a resolution in the order of magnitude of 1 meter. This increasing precision generates a significant amount of data. Techniques of automatic interpretation of remote sensing images which assign to each pixel a land cover class (road, water, ... ) become essential to process all data in reasonable time. In this paper, we present a novel approach to improve the automatic interpretation by combining several region-based classification methods.

For these images, objects of interest contain several pixels which are not easy to identify at pixel level [1], [2]. Generally a segmentation method is used to cluster pixels that belong to the same objects before a classification step, which is applied on resulting regions. For the classification step, many methods are available. We decide to focus on two major methods.

One of the most popular method for automatic image interpretation is supervised classification. The method consists in learning a classification algorithm which is able to identify classes of interest. Some regions which class is known are needed for this method. These samples are referred to as training set and are used for the learning step. When the learning step is completed, the induced algorithm is able to assign a class to each unlabelled region.

Knowledge-based object identification is another way to interpret images. We can use an ontology to represent geographical knowledge [3]. An ontology is a specification of an abstract, simplified view of the world that we wish to represent for some purpose [4]. The ontology defines a set of concepts (buildings, water, ...) and their relations. Each concept is defined by some low-level descriptors associated to intervals of accepted values. After an image segmentation, the segments are fed into a concept selection module using the ontology to select the most plausible concepts (i.e. classes). For this task, low-level descriptor values of the segments are computed and used for the matching tests with the descriptors of the concepts from the ontology.

These methods have pros and cons. Supervised classification is fast to realize and obtains good results if the training set is well constructed. Nevertheless, for certain classes, it is difficult to have enough samples to be exhaustive. The ontologybased approach profits from the knowledge of experts but totally depends on it. An ontology is descriptive and can have difficulties to distinguish some classes of objects if the concept descriptors overlap.

We experimented the proposed approach on an image corresponding to an urban district of Strasbourg, France, with a spatial resolution of 0.70 meters. The image is composed of four channels (blue, green, red and near infra-red). The resolution of the image is of $900 \times 900$ pixels. Some part of this image have been manually classified by an expert in three classes: road, vegetation and house. The image and samples can be viewed on Figure $1^{1}$.

In this paper, we propose a hybrid method combining an ontology-based approach with a supervised classification algorithm. The proposed approach takes advantage of the two classifiers using the most suitable one according to the class to recognize. The supervised classification is the default classifier. The ontology-based classifier is used for the classes not recognized by the supervised algorithm or if the user chooses to use it for some classes.

\section{OBJECTS SEGMENTATION AND CHARACTERIZATION}

The first step in object-based remote sensing image interpretation is to obtain a good segmentation. Many algorithms are available for remote sensing image segmentation [5], [6], [7], [8], [9], [10]. In this paper we use one of our methods [11]. This method takes advantage of pixel samples for some classes. In our case samples from houses, road and vegetation were used. A classical region merging algorithm were applied in order to obtain an even better segmentation with less regions. A part of the final segmentation is show on Figure

\footnotetext{
${ }^{1}$ All the images of the paper are available in colour at http://lsiit.ustrasbg.fr/afd/publications/igarss2007/.
} 


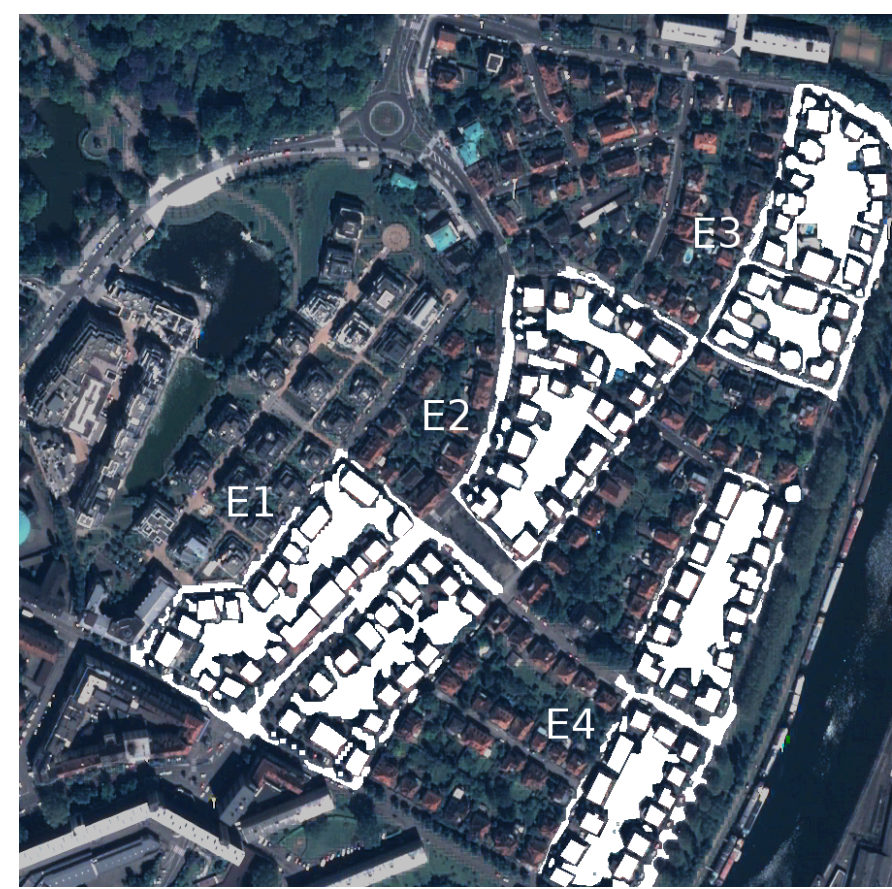

Fig. 1. Original image and pixels samples (in bright).

2. User should note that even without learning with samples from the water, the waterway on the right of the segmentation part is well segmented.

The next step when a segmentation of the image is obtained is to characterize each region. Many features are extracted, namely :

- spectral statistics: mean and standard deviation for each image channel.

- spectral indices: NDVI index (Normalized Difference Vegetation Index) and the SBI index (Soil Brightness Index).

- spatial features: area, elongation, diameter (length of the major axis), compactness (Miller index), solidity (ratio of the area to the convex hull area), etc.

\section{OBJECTS CLASSIFICATION WITH A $k$ NEAREST NEIGHBOUR CLASSIFIER}

When each region of the segmentation is characterized, the last step is to assign, to each region, a class label. A such objective can be achieve by supervised classification algorithm by example the $k$ nearest neighbour classifier [12]. A supervised classification algorithm work in a two step way. First, some regions for which the class label is know are given to the algorithm. At second step, the classifier can assign a class label to a region which class label is "unknown". This assignment is performed using case given in the first step. For an unlabelled region, the $k$ nearest labelled regions in feature space are selected. Each of these regions increases the membership for its class. Weight of each selected region is the inverse of the distance between itself and the pixel to classify in the feature space. If $x$ denotes the current region,

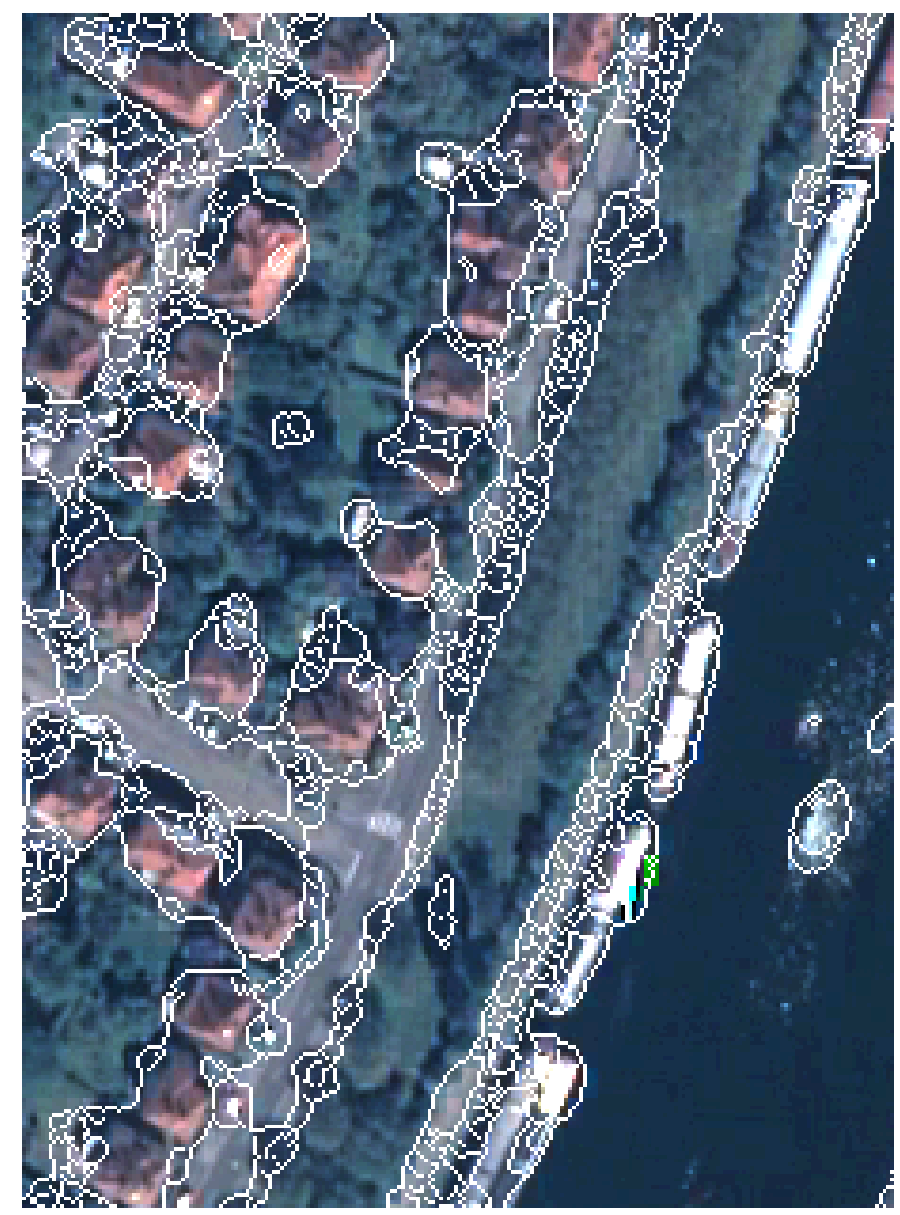

Fig. 2. Part of the obtained segmentation

$x_{n}$ for $n=1 \ldots k$ the five nearest regions in feature space and $d\left(x, x_{n}\right)$ the distance between $x$ and $x_{n}$, probability of membership to class $i$ (with $N C$ classes), namely $p_{x i}$, is obtained by :

$$
p_{x i}=\frac{1}{W} \sum_{n=0}^{k} w_{n i}
$$

where $w_{n i}= \begin{cases}d\left(x, x_{n}\right)^{-1} & \text { if } x_{n} \text { is labelled with class } i \\ 0 & \text { otherwise }\end{cases}$ and $W=\sum_{n=0}^{5} \sum_{i=1}^{N C} w_{n i}$

The region is finally labelled by the class $i$ for which $p_{x i}$ is the greater.

Samples are given before the segmentation step. Thus, the mapping between theses samples and obtained regions is not trivial. For example, if only one pixel is know to be a road pixel for a region with 100 pixels, this region should not be used as a learning region with class label road. The training regions are chosen using the following algorithm : for each produced region having $n$ pixels, if the number of labelled pixels of one class $c$ is greater than $n / 2$, then this regions is labelled by $c$. 


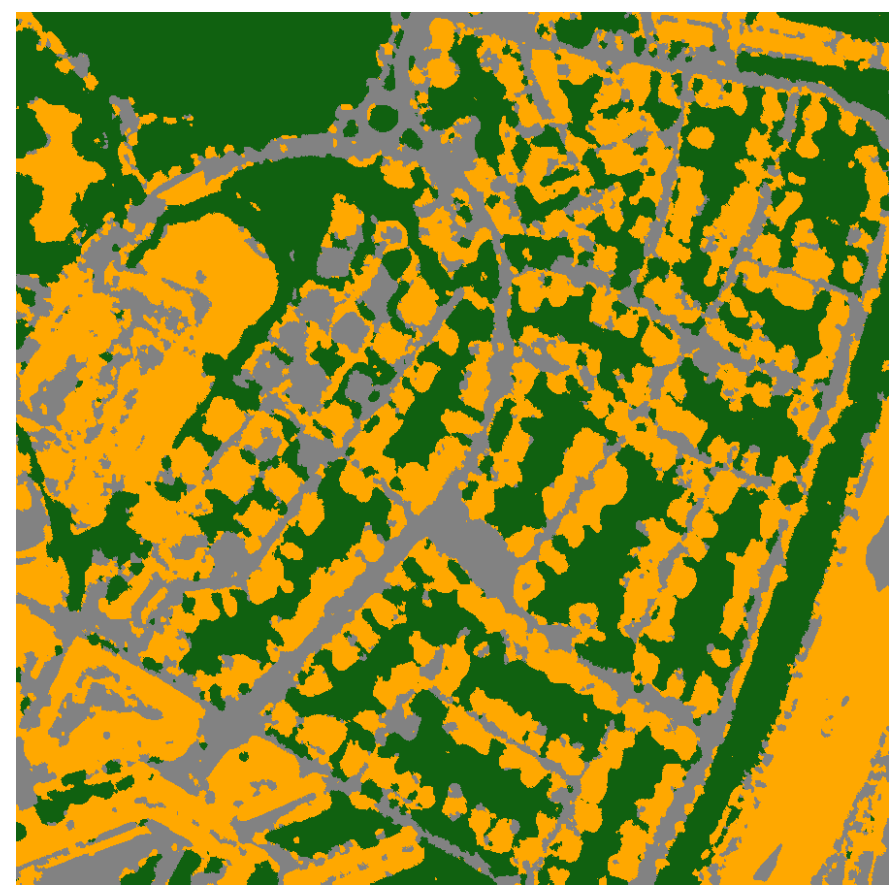

Fig. 3. Object-based classification with a 5 nearest neighbour classifier.

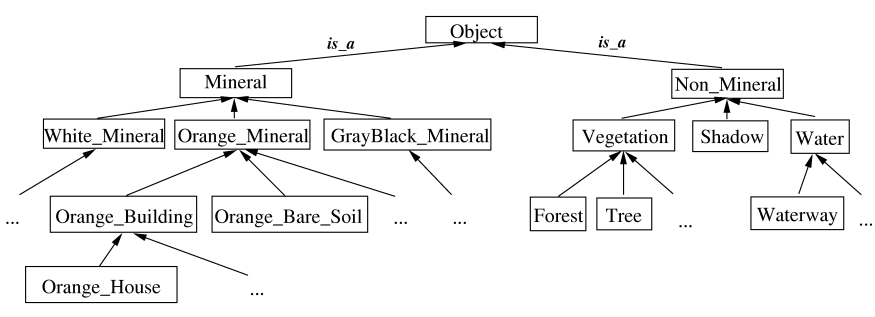

Fig. 4. Excerpt of the ontology.

Pixels samples used to induce the classifier can be found on Figure 1. Four areas $(E 1, \ldots$, E4) are classified by an expert. Three classes of interest was considered: house, vegetation, road. Pixels not labelled in these areas are unable to be classified because of mixed pixels or pixels of an irrelevant class.

The classification result is shown on Figure 3. A crossvalidation evaluation was used to assess the classification quality. Each labelled area is used as an evaluation area and the three others are used for learning. The average result is $89.85 \%$ of well-classified pixels.

\section{OBJECTS CLASSIFICATION WITH AN ONTOLOGY}

The regions and their features are the input the ontologybased object recognition. The method consists in matching each region with the concepts of an ontology. We have defined a matching measure and a traversing method of the ontology [3]. The used ontology (see Figure 4) is composed of 91 concepts, 20 attributes and 66 final concepts.

The proposed matching method is a feature-oriented approach. This corresponds to verify the validity of feature values of the region according to the properties and the constraints defined in the concepts. The measure is composed of a local component (dealing with the inner properties of the concept) and a global component (evaluating the pertinence in the hierarchy of concepts). The local similarity measure $(\operatorname{Sim}(R, C))$ compares the features of a region $R$ with the specific attributes of a concept $C . \alpha_{i}$ is the weight of $A_{i}$, expressing the role of $A_{i}$ to recognize $C$. Valid evaluates the validity of an extracted feature $v_{i}$ and the bounds of the accepted values of an attribute $A_{i}\left(\operatorname{Valid}\left(v_{i}, A_{i}\right)=1\right.$ if $v_{i}$ satisfies $\left.A_{i}\right)$. The matching score $(\operatorname{Score}(R, C))$ evaluates the pertinence of the matching between a region $R$ and a concept $C$ in the hierarchy of concepts. The matching score is a linear combination of local similarity measures obtained with the concepts $C_{j}$ of the path starting from the root of the ontology and ending at the studied concept $\left(C_{m}=C\right)$. The local similarities are propagated by inheritance to more specific concepts. In this computation, we integrated a specialization coefficient based on the depth $\beta_{j}$ of the concepts. In this way, the measure favours the specialization of the concepts, considering all additional information give a new semantic.

$$
\begin{gathered}
\operatorname{Sim}(R, C)=\frac{\sum_{i=1}^{n} \alpha_{i} \operatorname{Valid}\left(v_{i}, A_{i}\right)}{\sum_{i=1}^{n} \alpha_{i}} \\
\operatorname{Score}\left(R, C_{m}\right)=\frac{\sum_{j=1}^{m} \beta_{j} \operatorname{Sim}\left(R, C_{j}\right)}{\sum_{j=1}^{m} \beta_{j}}
\end{gathered}
$$

The matching score between a region and a concept being defined, it remains to traverse the ontology to find the best concept(s) according to the score for a region. We developed an level-wise algorithm to traverse the ontology using heuristics to reduce the search space. The general sketch of the exploration is: if the region matches the current concept, the algorithm will go deeper in the hierarchy in the next level. If matching fails, the current concept is dropped and its subconcepts will not be explored. The main heuristic corresponds to the selection of the best concepts at each level in order to prune some branches which the starting concept would not be relevant (with a poor matching score value). This strategy is based on the fact that an internal concept has properties more general than its children. If a few of these properties (or none) are valid, its child will not be relevant.

For the experiments, we used an excerpt of our ontology. Three classes are used: vegetation, water and towers. Let us note that if no label is found for a region, the system uses the label "unknown". The results are presented in Figure 5. Houses and road are not used because of the inability of the ontology to distinguish them. The oversegmentation leads the ontology to miss many road regions as elongation is not relevant for small regions and many road regions are recognized as houses.

\section{Merging Ontology And neARest neighbour CLASSIFIER RESULTS}

In the two previous sections, results of a supervised classification (Section III) and an ontology classification (Section IV). None of them gives a satisfying result. On the one hand, the supervised classification need a training set to learn how 


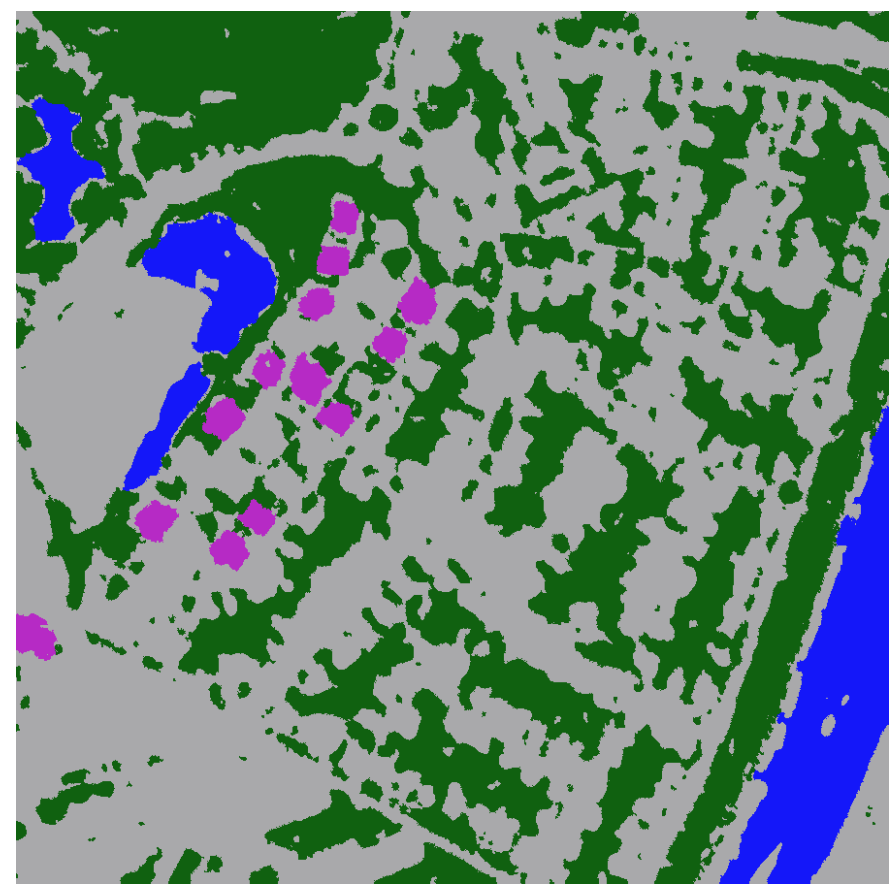

Fig. 5. Object-based classification with the ontology.

to distinguish classes. In our case only samples of houses, vegetation and road where available. Water and tower regions can not be detected by a such classifier. On the other hand, the ontology classifier has great difficulties to recognize road and houses (see Section IV).

To achieve better classification, we decide to combine these two methods. The supervised classifier gives an initial result. Each region is labelled with road, vegetation or house. Water and towers can not be identified. Then, this result is enhanced with some labels from the ontology classification, namely towers and water labels which replace labels given by the supervised classification. Vegetation classification quality is quite similar with the two classifiers. The final result is shown on Figure 6.

\section{CONCLUSION}

In this paper, we proposed to combine two different classification approaches: a supervised classification and an ontologybased classification. We shown that these two approaches are complementary and our result shows the relevance of our proposal.

Future works will include an automatic class selection process based on a collaboration of the different approaches. Another work will be to extend our approach to more classes.

\section{REFERENCES}

[1] L. Rego and B. Koch, "Automatic classification of land cover with high resolution data of the rio de janiero city brazil: Comparison between pixel and object classification," in Proc. the Symposium on Remote Sensing of Urban Areas, Regensburg, Germany, June 2003, pp. 16821777.

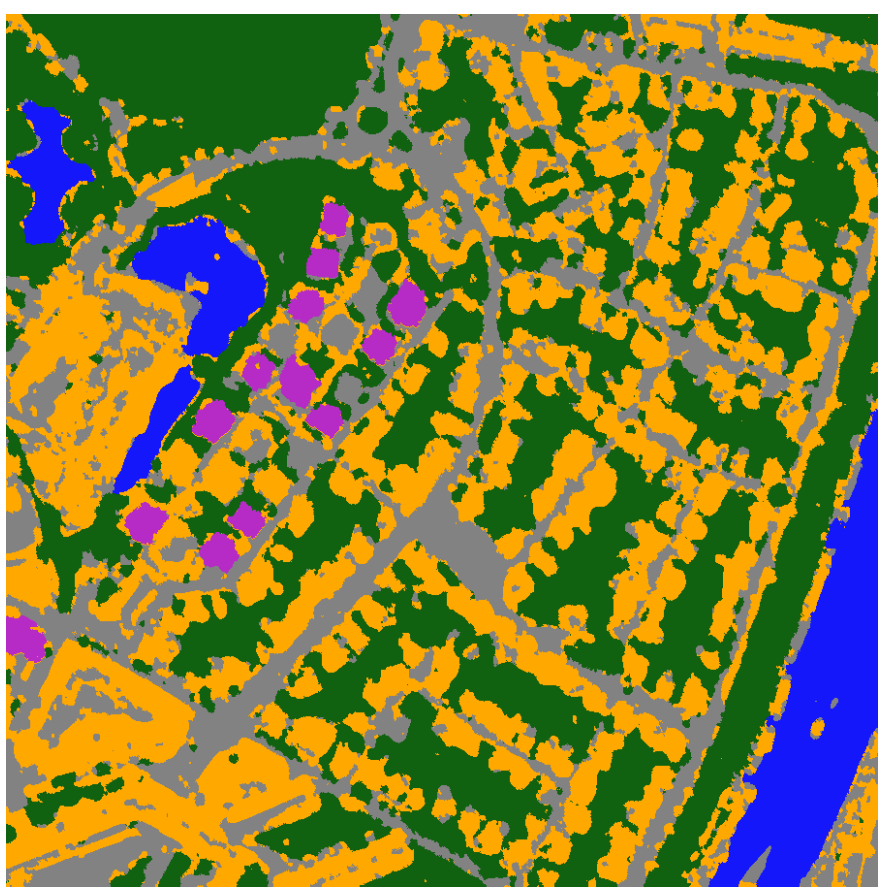

Fig. 6. Final results.

[2] T. Whiteside and W. Ahmad, "A comparison of object-oriented and pixel-based classification methods for mapping land cover in northern australia," in Proc. SSC 2005, Melbourne, Australia, September 2005, pp. $1225-1231$.

[3] N. Durand, S. Derivaux, C. Wemmert, and P. Gançarski, "Ontologybased object recognition for remote sensing image interpretation," Louis Pasteur University, LSIIT - AFD Team, Strasbourg, France, Tech. Rep., April 2007

[4] T. Gruber, "Toward principles for the design of ontologies used for knowledge sharing," International Journal of Human Computer Studies, vol. 43, no. 5/6, pp. 907-928, 1995.

[5] M. Baatz and A. Schape, "Multiresolution segmentation: an optimization approach for high quality multi-scale images segmentation," in Angewandte Geographische Informationsverarbeitung XI, 2000.

[6] A. P. Carleer, O. Debeir, and E. Wolff, "Assessement of very high spatial resolution satellite image segmentations," Photogrammetric Engineering and Remote Sensing, vol. 71, no. 11, pp. 1285-1294, 2005.

[7] M. Mueller, K. Segl, and H. Kaufmann, "Edge- and region-based segmentation technique for the extraction of large, man-madeobjects in high-resolution satellite imagery," Pattern Recognition, vol. 37, no. 8, pp. 1619-1628, 2004.

[8] N. Malpica, J. Ortuno, and A. Santos, "A multichannel watershed-based algorithm for supervised texture segmentation," Pattern Recognition Letters, vol. 9-10, no. 24, pp. 1545-1554, 2003.

[9] G. Castilla, "Size-contrained region merging: A new tool to derive basic landcover unit from remote sensing imagery," in Proc. ESA-EUSC 2004, 2004.

[10] Q. Chen, C. Zhou, J. Luo, and D. Ming, "Fast segmentation of highresolution satellite images using watershed transform combined with an efficient region merging approach.” in Proc. IWCIA, ser. LNCS, R. Klette and J. D. Zunic, Eds., vol. 3322. Springer, 2004, pp. 621-630.

[11] S. Derivaux, S. Lefèvre, C. Wemmert, and J. Korczak, "Watershed segmentation of remotely sensed images based on a supervised fuzzy pixel classification," in Proc. the IEEE International Geosciences And Remote Sensing Symposium (IGARSS), 2006.

[12] D. W. Aha, D. F. Kibler, and M. K. Albert, "Instance-based learning algorithms," Machine Learning, vol. 6, pp. 37-66, 1991. 